\title{
Parte 2: Manejo de la Alergia a la Proteína de Leche de Vaca.
}

\section{Part 2: Cow's Milk Protein Allergy Management.}

\author{
Norma González Hernández*, Susan Rodríguez González**, Lidia María Prado López ***, \\ Gabriela López****
}

\section{RESUMEN}

La lactancia materna es un excelente factor protector y una forma inigualable de facilitar el alimento ideal para el crecimiento y desarrollo saludable. Es imprescindible su recomendación exclusiva durante los primeros 6 meses de vida y de ser posible continuar con la lactancia materna durante la alimentación complementaria hasta los 2 años de edad; solo se debe recurrir a fórmulas especiales cuando la madre no pueda amamantar. Para estos casos se dispone de varias fórmulas hidrolizadas, soja y elementales.

Las fórmulas hidrolizadas pueden ser extensa o parcialmente hidrolizadas, y ser séricas o de caseína. Las fórmulas extensamente hidrolizadas, han sido sometidas a ensayos clínicos donde se comprueba su hipoalergenicidad al eliminar los síntomas de la Alergia a la Proteína de la Leche de Vaca (APLV) luego de su inicio. Estudios recientes demuestran que la adición de Lactobacillusr hamnosus (LGG) a una fórmula de caseína extensamente hidrolizada acelera la adquisición de tolerancia en estos niños en comparación con los pacientes que recibieron fórmula sin adición.

La fórmula de soja, aunque no es hipoalergénica, se puede emplear para tratar la APLV, pero no antes de los 6 meses. No se pueden emplear como tratamiento de APLV fórmulas parcialmente hidrolizadas, ni leche de otros rumian-

\footnotetext{
* Gastroenteróloga pediatra, Pediatra de turno Instituto Hondureño de Seguridad Social.

** Estudiante de 5 año Universidad Nacional Autónoma de Honduras en el Valle de Sula (UNAH-VS).

*** Pediatra de guarda HNMCR, Docente UNAH-VS.

**** Residente de segundo año, Posgrado de Pediatría, UNAH-VS.

Dirigir correspondencia a: christiandng@hotmail.com

Recibido: 28 de mayo $2017 \quad$ Aprobado: 12 de septiembre 2017
}

tes, como oveja o cabra. En pacientes con altos niveles de sensibilización o con antecedente de reacción anafiláctica, se recomienda la administración de fórmula elemental. El objetivo de la presente revisión es dar a conocer el manejo y uso racional de las fórmulas hidrolizadas y elementales en niños con APLV y así evitar el uso indiscriminado de estas en niños con trastornos funcionales.

\section{PALABRAS CLAVE}

Fórmulas infantiles, hidrolizados de proteína, Lactobacillus rhamnosus, leche de soja, aminoácidos.

\section{ABSTRACT}

Breastfeeding is an excellent protection factor for babies and it is an incomparable way of providing ideal food for healthy growth and development to a child. It is imperative to recommend its use exclusively during the first 6 months of age, and continue breastfeeding during complement feeding until 2 years of life. Special formulas should be used only when mothers cannot breastfeed. In this case scenario, there are several formulas available, such as hydrolyzed formulas, soy formulas and elemental formulas. Hydrolyzed formulas could be either extensively or partially hydrolyzed and could be serum protein or casein based. The extensively hydrolyzed formulas (eHF), have been proven to eliminate symptoms of Cow Milk Protein Allergy (CMA) in clinical trials. Recent studies have shown that the addition of Lactobacillus rhamnosus (LGG) to an eHFcasein formula, accelerates the acquisition of tolerance in children compared to patients receiving no LGG added eHF. 
Although soy formula is not hypoallergenic, it could be used to treat CMA. It should not be used before 6 months of age. Partially hydrolyzed formulas or milk from other ruminants, such as sheep or goat, can't be used as treatment CMA. In patients with high levels of sensitivity or with a history of anaphylactic reaction, administration of elemental formula is recommended.

The aim of this review is to inform the management of CMA,to recommend a wise use of hydrolyzed and elementary formulas and to advice the avoidance of the indiscriminate use of these formulas in children with functional disorders.

\section{KEYWORDS}

Infant formulas, soymilk, soy protein, ProteinHydrolysates, aminoacids, actobacillus rhamnosus.

\section{INTRODUCCIÓN}

La APLV es la alergia más común de la infancia, y es poco común en adultos. Comprende un gran espectro de síndromes debido a respuestas inmunológicas mediadas o no por lgE. La APLV no incluye otras reacciones a la leche como la intolerancia a la lactosa que no tiene reacción inmunológica.

Se revisa aquí aspectos sobre el manejo de APLV. Existen múltiples beneficios de la lactancia materna (LM) a corto y largo plazo en el niño y la madre. Sin embargo, la presente revisión se centra en la descripción de 3 fórmulas de sustitución disponibles en el mercado.

\section{LACTANCIA MATERNA EXCLUSIVA}

La leche materna es un alimento completo cuya composición es la ideal para el lactante, ya que proporciona todos los nutrientes y el agua necesaria durante los primeros 6 meses de vida y aporta el $50 \%$ de estos hasta el segundo año de vida.(1) Tiene propiedades inmunitarias que protegen al lactante creando una flora intesti- nal bifidogénicasaludable, ${ }^{(2)}$ que ayuda en la prevención de infecciones intestinales, disminuyendo la morbi-mortalidad en los primeros 2 años de vida. Además, dado las concentraciones adecuadas de ácidos grasos: DHA y ARA nos ayuda a mejorar la agudeza visual ${ }^{(3)}$ y el desarrollo cognitivo. ${ }^{(4)}$ La óptima calidad de las proteínas y sus aminoácidos reducen el stress metabólico, hepático y renal disminuyendo la sobrecarga renal de solutos y así mismo reduce el riesgo de sobrepeso, obesidad, ${ }^{(5)}$ cáncer, $^{\left({ }^{(6)}\right.}$ enfermedad coronaria ${ }^{(7)}$ y DM tipo 2 , tanto en países desarrollados como en los subdesarrollados. Amamantar inmediatamente después del parto favorece la "vinculación afectiva" entre madre e hijo, que tiene grandes ventajas emocionales ${ }^{(8)}$ y ayuda a asegurar el lugar del bebe dentro de la familia.

Cabe mencionar que también la madre obtiene beneficios como el espaciamiento de los nacimientos, evidenciado en que las madres que amamantan tienen un período de esterilidad mas largo después de dar a luz, ${ }^{(9)}$ reducción del riesgo de cáncer de mama ${ }^{(10)}$ y ovario, osteoporosis, ${ }^{(11)}$ enfermedad cardiaca vascular, hiperlipidemia, hipertensión arterial y Diabetes Mellitus tipo $2^{(12)}$, dicha reducción es proporcional al tiempo que la madre de lactancia.

La evidencia científica avala la superioridad de la leche materna para la alimentación del recién nacido y del lactante durante los primeros 6 meses de vida, periodo en el cual es indispensable. Se puede mantener hasta los 2 años. El uso de la LM prolongado es lo ideal en prevención y tratamiento de las reacciones adversas a la proteína de leche de vaca (PLV), por lo que es el PILAR FUNDAMENTAL de manejo de la APLV. ${ }^{(1,13)}$

Es importante recalcar que al recomendar la lactancia materna exclusiva (LME) a un paciente con APLV se debe instruir a la madre que debe evitar al máximo la ingesta de leche, deri- 
vados y productos que contengan PLV ocultas y debe recibir un suplemento de calcio: $1 \mathrm{~g} /$ día. Al prescribir dietas de eliminación se debe realizar como si fuera prescripción de medicamentos. Se le debe enseñar a la madre a escudriñar la etiqueta de los alimentos para detectar fuentes potenciales de alérgenos. En la etiqueta de alimentos se le designa a la leche como caseína, caseinato, suero/whey, lactoalbumina, turrón/nougat, sabor natural/natural flavoring, sabor caramelo/caramel flavoring, proteína hidrolizada. También está presente en productos no lácteos como cremas y quesos de soja, fiambre y hotdogs y no es poco común hallarla en postres congelados y de soja.. ${ }^{(14)}$

Se puede encontrar información sobre la dieta de eliminación en la APLV, con listado de alimentos permitidos y no permitidos en las siguientes páginas de internet: http://www. stanfordchildrens.org/en/topic/default?id=mil k-allergy-diet-for-children-90-P01696, https:// www.allergy.org.au/patients/food-allergy/a scia-dietary-avoidance-for-food-allergy .

\section{FÓRMULAS DE SUSTITUCIÓN EN APLV}

Los lactantes que no reciben lactancia materna deben recibir una fórmula de sustitución. Recuerde que se debe recurrir a fórmulas especiales únicamente en el caso que la madre no pueda dar pecho materno al bebe. En este caso, se dispone de varios tipos de fórmulas como las fórmulas hidrolizadas, a base de soja y fórmulas elementales a base de aminoácidos sintéticos. ${ }^{(15-17)}$

El cuidado que se debe tener con las fórmulas maternizadas es que el aporte nutritivo adecuado depende de la dilución. Al diluir inadecuadamente con exceso de agua las fórmulas maternizadas o al preparalas muy concentradas se reduce su valor nutritivo o no se proporciona la cantidad de agua necesaria. Además, se debe tener cuidado en NO DILUIRLAS CON AGUA CALIENTE ya que desnaturaliza las proteínas.
En esta sección se analizarán las fórmulas hidrolizadas, fórmulas a base de soya y fórmulas elementales.

\section{FÓRMULAS HIDROLIZADAS}

Para entender cómo las fórmulas utilizadas en el manejo de la APLV funcionan, se deben tomar en cuenta ciertos aspectos básicos de las particularidades de esta entidad nosológica.

Lo primero es que una característica importante de la PLV es que conserva su actividad alergénica aun después de bullirse, pasteurizarse y procesarse a ultra temperaturas o evaporarse. $^{(18)}$

Otro aspecto a recordar es el concepto de tolerancia y alergenicidad. La tolerancia se define como la ausencia de respuesta inmune frente a un antígeno inofensivo y se debe a una función adecuada de las células T reguladoras (Th3). La alergenicidad de una proteína es la capacidad que tiene una determinada molécula de desencadenar una reacción alérgica en individuos sensibles, y depende de tres factores: a) del peso molecular; por debajo de 2000 Daltons es raro que desencadene reacciones alérgicas, aunque no las descarta. B) la secuencia de aminoácidos y c) la configuración de la proteína en el espacio. ${ }^{(18)}$

El tercer aspecto a tomar en cuenta es que en la alergia la secuencia de aminoácidos, que recibe el nombre de epítope, es reconocida como antígeno por el sistema inmunológico. Existen 2 tipos de epítopes; los secuenciales, con estructura lineal y los conformacionales con estructura no lineal o tridimensionales. ${ }^{(19)}$ (Ver figura 1). 
Gráfico No. 1: Esquematización de los tipos de epítopes en la Proteína de la leche.

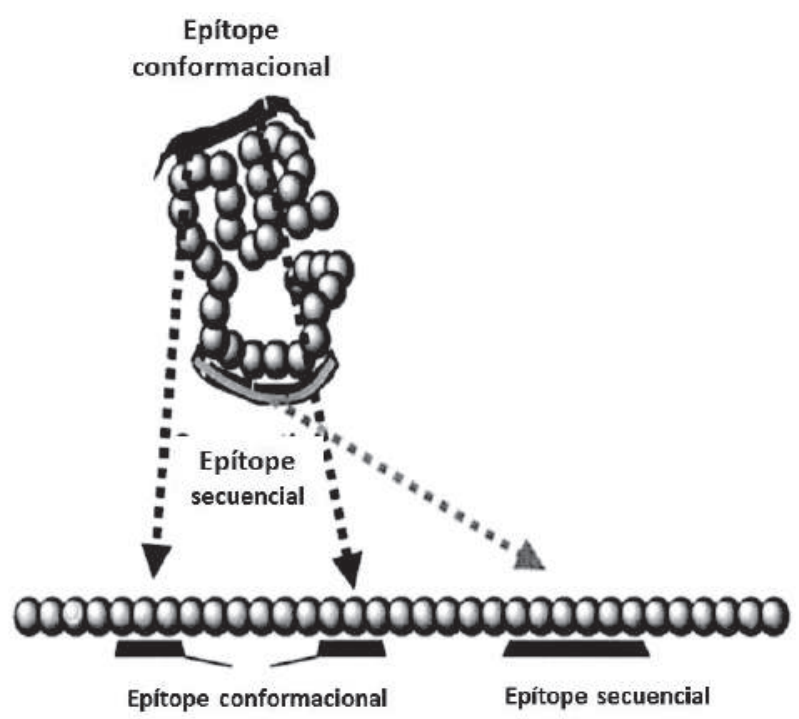

Fuente: Sampson, et al. Foodallergy: a practiceparameter update-2014. ${ }^{(19)}$

Es por esto que para disminuir la alergenicidad de la PLV, las proteínas en las fórmulas hidrolizadas son pre digeridas mediante hidrólisis a través de 3 técnicas: termólisis, hidrólisis enzimática y una combinación de ambas. ${ }^{(20)}$

Mediante hidrólisis térmica de la proteína se rompen los enlaces químicos, perdiendo así la estructura espacial. De esta forma se separan los epítopes conformacionales, manteniéndose la configuración lineal y disminuyendo su capacidad antigénica. ${ }^{(20)}$ Mediante la hidrólisis enzimática, se produce hidrólisis de los enlaces entre aminoácidos dividiéndose la proteína en fragmentos más pequeños. De esta forma se disminuye el peso molecular de la proteína, la carga antigénica de los epítopes secuenciales, y además facilita su digestión y absorción. ${ }^{(20)}$ En el tratamiento mixto (térmico y enzimático) de la proteína, se realiza un ultra filtrado para eliminar los fragmentos proteicos de mayor tamaño que no han perdido la capacidad antigénica, para conseguir la reducción de algunos aminoácidos que confieren mal gusto y los restos de las enzimas empleadas. ${ }^{(18,20)}$
El último aspecto a recalcar es que se considera que una fórmula es hipoalergénica cuando ha demostrado en estudios clínicos con diseño doble ciego control placebo y una confiablidad de $95 \%$ que no produce reacciones en $90 \%$ de los niños con APLV documentada. ${ }^{(21)}$

Las Fórmulas Hidrolizadas (FH) se distinguen según el grado de hidrólisis en extensa o parcialmente hidrolizadas y por la fuente de proteínas en séricas o de caseína. ${ }^{(22-23)}$

\section{Las Fórmulas Parcialmente Hidrolizadas} (FPH) (Con bajo grado de hidrólisis) son comercializados con las iniciales HA que se refiere a fórmulas hipoantigéni caso parcialmente hidrolizadas. Con la hidrólisis se obtiene naminoácidos, péptidos de diversos tamaños y menos del $1 \%$ de proteína intacta. El peso molecular de la proteína hidrolizada se encuentra entre 5,000 a 12,000 daltons ( $\mathrm{Da}$ ). Lo importante de recordar sobre las FPH es que nunca deben emplearse para el tratamiento de lactantes con APLV, ya que sus proteínas no presentan un grado de hidrólisis suficiente para evitar una respuesta alérgica. ${ }^{(13.28,20)}$

Estas fórmulas también son procesadas con termólisis e hidrólisis enzimática y posteriormente ultrafiltradas, obteniendo oligopéptidos y aminoácidos. Con esta modificación se consigue una menor antigenicidad, pero no implica nula alergenicidad, ya que péptidos de bajo peso molecular pueden conservar epítopes responsables de reacciones alérgicas a las PLV.

El Comité de Nutrición de la Sociedad Francesa de Pediatría no aconseja el uso indiscriminado de estas fórmulas, e insiste también en la superioridad de la lactancia materna para el tratamiento de los lactantes de riesgo atópico. ${ }^{(24)}$

Las Fórmulas Extensamente Hidrolizadas (FEH) (Con alto grado de hidrólisis) son fórmulas oligoméricas, también llamadas hipoalergénicas. 
Las FEH son más caras, con sabor y olor desagradables, y rara vez producen reacciones alérgicas. Estas fórmulas han sido sometidas a distintos ensayos clínicos donde se comprueba su hipoalergenicidad al demostrar que elimina los síntomas de la APLV. ${ }^{(25)}$

Se debe recordar que la respuesta inmunológica desencadenada en la ALPV depende básicamente de 2 mecanismos: mediada por lgE y no mediada por lgE. Las reacciones mediadas por lg E son disparadas por proteínas de más de 12 aminoácidos de longitud, los cuales tienen epítopes conformacionales. Las reacciones no mediadas por lgE son disparadas por epítopes en las células T que cubren de 6-12 aminoácidos en longitud. La hidrólisis extensa produce proteínas de un máximo de 2 a 3 aminoácidos. En principio, a mayor hidrólisis, menor longitud de cadena. Esa longitud se muestra en el perfil de peso molecular de la fórmula. ${ }^{(26)}$

La Academia Americana de Pediatría (AAP) considera una fórmula como extensamente hidrolizada si el peso molecular de los péptidos residuales es menor a 1,500 Da. Cuanto mayor es el grado de hidrólisis, menor es el peso molecular y existe menor capacidad antigénica. ${ }^{(1,27)}$

Todas las FEH tienen cierto potencial alergénico residual por la presencia de epítopes de 3-6 aminoácidos que pueden inducir, en el lactante muy sensibilizado, síntomas fundamentalmente gastrointestinales, y/o reacciones de alergia inmediatas. Es por esto que puede haber fracasos en el tratamiento en el $10 \%$ de los casos. En el caso de considerar la utilización de una FEH en este tipo de pacientes se debe tener la precaución de realizar el prick test y test de provocación oral previo al inicio de la $\mathrm{FEH},{ }^{(13,15,18,28)}$ o iniciar una fórmula elemental.

Las nuevas directrices sobre el enfoque terapéutico óptimo para niños con APLV, recomiendan el uso de fórmulas sustitutivas y demuestran que la adición del probióticoLactobacillusr hamnosus (LGG) a una fórmula de caseína extensamente hidrolizada, acelera la adquisición de tolerancia en estos niños en comparación con los pacientes que recibieron esta fórmula sola. ${ }^{(29-33)}$

La FDA reconoce al LGG como seguro para usarse en fórmulas infantiles. ${ }^{(34-37)}$ Antes de exponer los aspectos en los que el LGG ha demostrado ser beneficioso en el manejo de la ALPV se describirá brevemente los beneficios en general de los probióticos.

Los probióticos afectan el ecosistema intestinal estimulando los mecanismos inmunitarios y no inmunitarios de la mucosa a través de antagonismo y competencia con patógenos potenciales. Estos ejercen su acción in situ ya que compiten con los microorganismos patógenos por los nutrientes; generan hidrólisis de toxinas e inducen la producción de sustancias antimicrobianas, que incluyen péptidos de la respuesta inmune natural, inducen la producción de ácidos grasos de cadena corta, y ejercen acción antiinflamatoria local selectiva sobre los mecanismos intracelulares que participan en la replicación viral y tienen la capacidad de modificar la adhesividad bacteriana. ${ }^{(38)}$ Las funciones de los probióticos se resumen en las siguientes: a) Promueven la función de la barrera intestinal estabilizando su microflora, normalizando la permeabilidad intestinal aumentada y disminuyendo la absorción de antígeno, b) Como inmunomoduladores inducen la producción de ácidos orgánicos y la modulación en la síntesis de óxido nítrico. Estimulan la respuesta inmunológica y promueven inmunotolerancia a antígenos alimentarios, disminuyendo la respuesta alérgica a ciertos antígenos ${ }^{(37)}$ y c) Función antibacteriana; estimulan la secreción de mucina intestinal disminuyendo o bloqueando la adhesión de bacterias al epitelio o mediante la producción de sustancias Ilamadas defensinas. ${ }^{(38)}$ 
Los beneficios que se han encontrado con el uso de una FEH de caseína más LGG son la disminución de la inflamación de la mucosa colónica, con un descenso en la calprotectina fecal en infantes menores de un mes, ${ }^{(32)}$ disminuye la gravedad de la dermatitis atópica, inflamación intestinal y confiere mayor tolerancia en niños con APLV. ${ }^{(32-37)}$

La adquisición de tolerancia a la PLV, se debe a la función inmunorreguladora del LGG, que regula la generación de citoquinas (IL-4, IL-5, IL-10, IFN g, TGF $\beta$, y TNF $\alpha$ ), implicadas en la APLV mediada y no mediada por lgE que contribuyen en la regulación negativa del proceso inflamatorio. ${ }^{(30)}$

Los niños afectados por APLV que reciben FEH de caseína sola o combinada con LGG para el tratamiento dietético de su condición, logran tolerancia a los 12 meses significativamente con más frecuencia que los que reciben FHR, fórmulas de soja y fórmulas elementales, esto se observa tanto en la APLV mediada como en la no mediada por lgE. ${ }^{(29,39)}$

Las FEH con LGG reducen la incidencia de otras manifestaciones de tipo alérgico y aceleran el desarrollo de tolerancia oral en niños con APLV mediada por lgE. ${ }^{(40)}$

El uso de probióticos está contraindicado de forma absoluta en caso de septicemia, uso de catéter venoso central, inmunosupresión y niños con intestino corto por el riesgo de infección ya que se ha documentado el desarrollo de infecciones por bacterias y levaduras en dichas situaciones. El comité de la ESPGHAN y NASPGHAN analizó esta situación y en su escrito sobre las recomendaciones para el uso de fórmulas suplementadas con probióticos concluyó que el uso de probióticos está determinado por una nutrición adecuada, los beneficios y por la seguridad al administrar los probióticos. Existe evidencia documentada en estudios doble ciego caso control de que el uso de fórmulas suplementadas con probióticos en recién nacidos y menores de 4 meses de edad no desencadenan efectos adversos ni problemas en el crecimiento, por lo que se recomienda su uso en lactantes sanos. ${ }^{(29,41)}$

En general, la mayor evidencia clínica para el uso de probióticos está vinculada al mejoramiento de la flora intestinal y en la estimulación de la función inmunitaria. ${ }^{(42-43)}$

\section{FÓRMULA DE PROTEINA DE SOJA}

En estos preparados, el componente proteíco se obtiene de la proteína purificada de la soja. La soja pertenece a la familia de las leguminosas, la cual es tratada posteriormente con hidrólisis térmica para reducir la actividad de los inhibidores de tripsina y hemaglutininas. La reactividad cruzada entre PLV y proteína de soja se observa en el $70-80 \%$ delos casos. ${ }^{(25)}$

Las Fórmulas de Soya (FS) son fórmulas más baratas y con mejor sabor que la FEH. Las necesidades nutritivas están bien cubiertas en el niño y en el adulto, pero no en el recién nacido, por lo que el Comité de Nutrición de la ESPGHAN y la AAP han definido recomendaciones sobre la composición de estas fórmulas, que deben ser suplementadas con aminoácidos azufrados (L- metionina y L-Carnitina), de las que la soja es carente, y enriquecidas con hierro y zinc, debido a su baja biodisponibilidad por el alto contenido en fitatos que quelan estos micronutrientes. ${ }^{(44-45)}$

Estas pueden utilizarse en el tratamiento de la APLV en todas sus manifestaciones, excepto en lactantes menores de 6 meses debido a sus riesgos nutricionales ya que se asocian con reducción de la absorción de minerales y microelementos. Además, contienen niveles altos de isoflavonas que tienen efecto estrogénico. No está indicada en enteropatía o malabsorción y tampoco está indicada con fines 
preventivos. La FS no es hipoalergénica ya que se ha documentado alergia en 10 a $50 \%$ de los pacientes. $^{(46-47)}$

La FS tiene indicaciones precisas como son la galactosemia (clásica y no clásica), en hijos de padres vegetarianos y es una opción alternativa en pacientes con APLV mayores de 6 meses, en los cuales se usa en caso de no contar con presupuesto necesario para el uso de una $\mathrm{FEH}$ en madres que no puede dar LM.

La FS está contraindicada en pacientes con insuficiencia renal y en pacientes con alteraciones metabólicas en relación a calcio y aluminio. Al utilizar una FS se debe suplementar calcio.

\section{FÓRMULA DE AMINOÁCIDOS}

La fórmula con aminoácidos (FAA) es la última opción terapéutica. Con esta fórmula no existe riesgo de reacción adversa. Esta contiene una mezcla compuesta $100 \%$ por aminoácidos libres, polímeros de glucosa y aceites vegetales, y al no contener péptidos poseen menor potencial de alergenicidad. ${ }^{(47)}$

Sus inconvenientes principales son el precio y el sabor. Se emplean en el lactante que no tolera-ninguno de los preparados anteriores, en reacciones anafilácticas graves, en alergias alimentarias múltiples y diarreas intratables. ${ }^{(1,18,25)}$

Las distintas entidades internacionales que se han pronunciado sobre el manejo de la APLV dan recomendaciones variables en cuanto a su indicación. La AAP, la Organización Mundial de
Alergias (WAO) y la ESPGHAN recomiendan el uso de la FAA como segunda opción para los lactantes en quienes los síntomas alérgicos persisten a pesar de usar una FEH. Además, la ESPGHAN lo aconseja como primera opción para lactantes con reacción anafiláctica severa, y en lactantes con enteropatía severa evidenciada por hipoalbuminemia y retraso del crecimiento. La WAO indica como primera opción para lactantes con anafilaxia, esofagitis alérgica eosinofílica y en la enfermedad pulmonar crónica inducida por PLV. ${ }^{(48)}$

Se debe mantener la dieta de eliminación de PLV, usando una fórmula hipoalergénica por lo menos durante seis meses o hasta los 9-12 meses de edad. Sin embargo, los lactantes con reacciones severas a la PLV pueden requerir una mayor duración de la dieta de eliminación, generalmente entre 12 y 18 meses, antes de repetir la prueba de provocación, previa prueba de lgE específica negativa. ${ }^{(48)}$

\section{CONCLUSIONES}

El pilar fundamental en el manejo de APLV, es la lactancia materna exclusiva siempre que sea posible, ya que se convierte en un excelente factor protector. Como opción alternativa de manejo se tienen las FEH de caseína o proteína de suero. Con la reciente adición de LGG a una FEH de caseína se ha logrado acelerar la adquisición de tolerancia a la PLV.

Las FPH nunca deben emplearse en APLV, ya que sus proteínas no presentan un grado de hidrólisis suficiente para evitar una respuesta alérgica. 


\section{BIBLIOGRAFÍA}

1. Lapeña López de Armentia S, Naranjo Vivas D. Alergia a proteína de leche de vaca. Pediatr Integral [ internet]. 2013 [citada en 19 de octubre 2015];17(8): 554-563. Disponible en: http://www.pediatriaintegral.es/ wp-content/uploads/2013/xvii08/02/ 554-563\%20Alergia\%20vaca.pdf.

2. Beattie LM, Weaver LT. Mothers, babies and friendly bacteria. Arch Dis Child Fetal Neonatal. 2011; 96 (3): F160-3.

3. Anderson GJ, Connor WE, Corliss JD. Docosahexaenoic acid is the preferred diatery $n 3$ fatty acid for the development of brain and retina. Pediatr Res 1990; 27(1): 89-97.

4. Kramer MS, Aboud F, Mironova E, Vanilovich I, Platt RW, Matush $L$, et al. Breastfee ding and child cognitive development: new evidence from large randomized trial. Arch Gen Psychiatry. 2008; 65(5): 578-584.

5. Gillman MW, Rifas-Shiman SL, Camargo CA, Berkey CS, Frazier AL, Rockett HR, et al. Risk of overweight among adolescents who were breastfed as infants. JAMA. 2001; 285(19): 2461-2467.

6. Kries R, Koletzko B, Sauerwald T, Mutius E, Barnert D, Grunert V, et al. Breast-feeding and obesity: cross sectional study. BMJ. 1999; 319 (7203): 147-150.

7. Owen CG, Whincup PH, Odoki K, Gilg JA, Cook DG. Infant feeding and blood colesterol: a Study in adolescents and a systematic review. Pediatrics 2002; 110 (3): 597-608.

8. Heikkilä K, Sacker A, Kelly Y, Renfrew MJ, Quigley MA. Breast feeding and child behaviour in the millennium cohort study. Arch Dis Child 2011; 96(7): 635-642.
9. Campbell OM, Gray RH, Characteristics and determinants of postpartum ovarian function in women in the United States. Am J ObstetGynecol 1993; 169(1): 55-60.

10. Collaborative Group on Hormonal Factors in Breast Cancer. Breast Cancer and Breastfeeding: Collaborative reanalysis of individual data from 47 epidemiological studies in 30 countries including 50302 women with breast cancer and 96973 women without the disease. Lancet. 2002; 360 (9328): 187-95.

11. Chantry CJ, Avinger P, Byrd RS. Lactation among adolescent mothers and subsequent bone mineral density. Arch PediatrAdolesc Med. 2004; 158(7): 650-6.

12. Schwarz EB, Ray RM, Stuebe Am, Allison MA, Ness RB, Freiberg MS et al. Duration of lactation and risk factor for maternal cardiovascular disease. Obstet Gynecol. 2009; 113(5): 974-82.

13. de Goicoechea Manzanares E, Torres Peral R, Lorente Toledano F. Guía para el tratamiento de lactantes con alergia a proteínas de leche de vaca. BOL PEDIATR [Internet]. 2009 [citada el 13 de noviembre del 2015]; 49(207): 3-15. Disponible en: https://www. sccalp.org/documents/0000/0731/BolPe diatr2009_49_003-015.pdf

14. Sampson HA. Food allergy. Part 2: diagnosis and management. J Allergy Clinlmmunol. 1999; 103(6):981-989.

15. Plaza Martin, AM. Alergia a proteína de leche de vaca. Protocdiagn ter pediatri.[Internet]. 2013[citada el 13 de noviembre del 2015];1: 51-61. Disponible en: http://www. aeped.es/sites/default/files/documen tos/5-aplv.pdf. 
16. Centro Nacional Excelencia Tecnológica en Salud de Mexico. Guía práctica clínica del manejo de la alergia a la proteína de leche de vaca [Internet]. México D.F.: CENETEC; 2011 [citada en 19 de octubre 2015]. Disponible en: http://www.cenetec.salud.gob.mx /descargas/gpc/CatalogoMaestro/502_GP C_Aletrgia_aleche_de_vaca/GER_Alergia LecheVaca.pdf.

17. Caballero T. Alergia a la Proteina de leche de vaca. En:Boyano M, Caballero T, Diaz A, García M, Pedrosa M, Quirce S. Alergia a Alimentos en el niño. España: MRA; 2013. Pp. 29-39.

18. Spieldennera J, Bellib D, Dupontc C, Haschkea F, Iskedjiand M, Nevot S, et. Al. La fórmula para el lactante basada 100\% en suero de leche parcialmente hidrolizada y la prevención de la dermatitis atópica: análisis farmacoeconómicos comparativos. Ann NutrMetab. 2011; 58 (supl.1): 44-52.

19. Sampson HA, Aceves S, Bock SA, James J, Jones S, Lang $D$, et al. Food allergy: a practice parameter update-2014. J Allergy ClinImmunol. 2014; 134(5): 1016-25. e43.

20. Fiocchi A,Brozek J, Schünemann $H$, BahnaS, von Berg A, Beyer K, et al.World Allergy Organization (WAO) Diagnosis and Rationale for Action against Cow's Milk Allergy (DRACMA) Guidelines. World Allergy Organ J. 2010; 3(4):57-161.

21. Moreno García L. Alergia a las proteínas de leche de vaca. Bol. SPAO [Revista en internet]. 2010 [citado 20 oct 2015]; 4(2): 55-66. Disponible en: https://www.spao.es/ima ges/publicaciones/boletinesSPAO/pdf/bo letin2-2010.pdf.
22. Lambers T, Gloerich J, van Hoffen $\mathrm{E}$, Alkema W, Hondmann D, van Tol EA. Clustering analyses in peptidomics revealed that peptide profiles of infant formulae are descrip- tive. Food Science \& Nutrition. 2015; 3(1): 81-90.

23. Pedrón-Giner C, Moreno-Villares JM,Dalmau-Serra J, Comité de Nutrición de la Asociación Española de Pediatría. Fórmulas de nutrición enteral en pediatría. AnPediatrContin. [Internet]. 2011 [citado 30 ene 2016];9(4):209-23 Disponible en: http:// www.aeped.es/sites/default/files/docu men tos/v9n4a526pdf001.pdf.

24. Tamayo López G,Sáenz de Urturi A,Hernández Sáez MR,Pedrón Giner C, García Novo M. Fórmulas infantiles especiales. AnEspPe diatr. 1997; 47(5):455-465.

25. Berni Canani R, Nocerino R, Terrin G, Frediani T, Lucarelli S, Cosenza $L$, et al. Formula selection for management of children with cow's milk allergy influences the rate of acquisition of tolerance: A prospective multicenter study. J Pediatr. 2013; 163 (3): 771 $-7$.

26. Herz U. Immunological basis and management of food allergy. J PediatrGastroenterolNutr. 2008; 47(supl.2):S54-7.

27. Montijo Barrios E, López Ugalde MV, Ramírez Mayans JA, Anaya Flores MS, Arredondo García JL, Azevedo Tenorio I, et al. Guía latinoamericana para el diagnóstico y tratamiento de alergia a las proteínas de leche de vaca (GL-APLV). RevInvClín. [Internet]. 2014[citado 2016 sep. 28]; 66(supl.2): S9-S72. Disponible en: http://www.imbio med.com.mx/1/1/articulos.php?metho d=showDetail\&id_articulo=102542\&id _seccion=260\&id_ejemplar $=9968 \&$ id_re vista $=2$. 
28. Orsi M, Fernández A, Follett FR, Marchisone S, Saieg G, Busoni VB, et al . Alergia a la proteína de la leche de vaca: propuesta de guía para el manejo de los niños con alergia a la proteína de la leche de vaca. Arch. argent. pediatr [Internet]. 2009 [citado 2016 sep 28]; 107(5): 459-470. Disponible en: http://www.scielo.org.ar/scielo.php? script $=$ sci_arttext\&pid=S0325-0075200 9000500016\&lng=es.

29. BerniCanani R, Nocerino R, Terrin G, Coruzzo $A$, Cosenza $L$, Leone $L$, et al. effect of lactobacillus $G G$ on tolerance acquisition in infants with cow's milk allergy: A randomized trial. J Allergy Clinlnmunol. 2012; 129(2): 580-2.

30. Canani RB, Di Costanzo M. Gut Microbiota as potential therapeutic target for the treatment of cow's milk allergy. Nutrients. 2013; 5(3): 651-62.

31. Scalabrin DM, Johnston WH, Hoffman DR, P'Pool VL, Harris CL, MitmesserSH.Growth and tolerance of healthy term infants receiving hydrolyzed infant formulas supplemented with Lactobacillus rhamnosus GG: randomized, double blind, contrelled trial. ClinPediatr (Phila). 2009; 48(7):734-44.

32. Nermes M, Kantele JM, Atosuo TJ, Salminen $\mathrm{S}$, Isolauri E. Interaction of orally administered Lactobacillus Rhamnosus GG whin skin and gut microbiota and humural immunity in infants with atopic dermatitis. ClinExp Allergy. 2011; 41(3): 370-377.

33. Baldassarre $M E$, Laforgia $N$, Fanelli $M$, Laneve A, Grosso R, Lifschitz C. LactobaciIlus GG Improves Recovery in Infants with Blood in the Stools andPresumptive Allergic Colitis Compared with Extensively Hydrolyzed formula alone. J Pediatr. 2010; 156(3): 397-401.
34. Muraro A, Hoekstra MO, Meijer Y, Lifschitz C, Wampler JL, Harris C, et al. Extensively hydrolysed casein formula supplemented with Lactobacillus Rhamnosus GG maintains hypoallergenic status: randomissed double-blind, placebo-controlled crossover trial. BMJ Open. 2012; 2(2): e000637.

35. Martin R. Generally Recognized as safe (GRAS) Determination for the use of Lactobacillus rhamnosus Stain HN001 in conventional foods. NewZealand: J Heimbach LLC. 2009.

36. European Food Safety Authority (EFSA). Scientific Opinion of the Panel on Biological Hazards on a request from EFSA. The maintenance of the list of QPS microorganisms intentionally added to food or feed. The EFSA Journal. 2008; 6(12): 1-923.

37. WorldGastroenterologyOrganization (WGO).[internet] Guías Mundiales de la Organización Mundial de Gastroenterología: probióticos y prebióticos. USA; wdo; 2017. [2015 enero 16]. Disponible en: http://www.worldgastroenterology.org/ guidelines/global-guidelines/probioti cs-and-prebiotics/probiotics-and-prebioti cs-spanish.

38. Thomas D, Greer FThomas DW, Greer FR; American Academy of Pediatrics Committee on Nutrition; American Academy of Pediatrics Section on Gastroenterology, Hepatology, and Nutrition. Probiotics and Prebiotics in pediatrics. Pediatrics. 2010; 126(6): 1217-1231.

39. Petschow BW, Figueroa R, Harris CL, Beck LB, Ziegler E, Goldin B. Effects of feeding and infant formula containing lactobacillus GG on the colonization of the intestine. J ClinGastroenterol. 2005; 39(9): 786-90. 
40. BerniCanani R, Di Costanzo M, Bedogni G, Amoroso A, Cosenza L, Di Scala C, Granata V, Nocerino R, Extensively hydrolysed casein formula containing L. rhamnosus GG reduces the occurrence of other allergic manifestations in children with cow's milk allergy: 3-year randomized controlled trial, Journal of Allergy and Clinical Immunology (2017), doi: 10.1016/j.jaci.2016.10.050.

41. Lifschitz C. Diarrea y probióticos. En: Larrosa-Haro A, Garibay ME. Nutrición y enfermedades del aparato digestivo en niños. 2 ed. Mexico: NestleNutritionInstitute; 2011. p.23-44.

42. Floch $\mathrm{MH}$, Walker WA, Madsen $\mathrm{K}$, Sanders ME, Macfarlane GT, Flint HJ, et al. Recommendations for probiotics use- 2011 update. J ClinGastroenterol. 2011; 45: S168-71.

43. Braegger C, Chmielewska A, Decsi T, Kolacek S, Mihatsch W, Moreno L, et al. Supplementation of infant formula with probiotics and/or prebiotics: a systematic review and comment by the ESPGHAN committee on nutrition. J PediatrGastroenterolNutr. 2011; 52(2):238-50.

44. Feferbaum R, Miuki C, Jacob A, Zamberlan P, Fuchs TV. fórmulas elementales y semi-elementales en pediatría. RevMexPediat [Internet]. 2010 [citado 20 dic 2015]; 77(4):164-171. Disponible en: http://www.medigraphic.com/pdfs/pediat/sp-2010/sp 104g.pdf.
45. Vandenplas $Y$, Koletzko $S$, Isolauri E, Hill D, Oranje AP, Brueton M, et al. Guidelines for the diagnosis and management of cow's milk protein allergy in infants. Arch Dis Child 2007; 92(10): 902-908.

46. Solinas C, Corpino M, Maccioni R, Pelosi U. Cow's milk protein allergy. J Matern Fetal Neonatal Med. 2010; 23(suppl 3): 76-79.

47. De Greef E, Hauser B, Devreker T, Veereman-Wauters G, Vandenplas Y. Diagnosis and management of cow's milk protein allergy in infants. World J Pediatr. 2012; 8(1): 19-24.

48. Koletzko S, Niggemann B, Arato A, Dias JA, Heuschkel R, Husby $S$, et al. Diagnostic approach and management of cow's milk protein allergy in infants and children: ESPGHAN GI committee practical guidellines. J PediatrGastroenterolNutr. 2012; 55(2): 221-229. 